\title{
Evidence of enrichment by individual SN from elemental abundance ratios in the very metal-poor dSph galaxy Boötes I
}

\author{
S. Feltzing ${ }^{1}$, K. Eriksson ${ }^{2}$, J. Kleyna ${ }^{3}$, and M. I. Wilkinson ${ }^{4}$ \\ 1 Lund Observatory, Box 43, 22100 Lund, Sweden \\ e-mail: sofia@astro.lu.se \\ 2 Department of Astronomy and Space Physics, Uppsala University, Box 515, 75120 Uppsala, Sweden \\ e-mail: kjell.eriksson@astro.uu.se \\ Institute for Astronomy, Honululu, 2680 Woodlawn Drive, Honolulu, HI 96822, USA \\ 4 Department of Physics and Astronomy, University of Leicester, University Road, Leicester LE1 7RH, UK \\ e-mail: miw6@astro.le.ac.uk
}

Received 6 July 2009 / Accepted 18 September 2009

\begin{abstract}
Aims. We establish the mean metallicity from high-resolution spectroscopy for the recently found dwarf spheroidal galaxy Boötes I and test whether it is a common feature for ultra-faint dwarf spheroidal galaxies to show signs of inhomogeneous chemical evolution (e.g. as found in the Hercules dwarf spheroidal galaxy).

Methods. We analyse high-resolution, moderate signal-to-noise spectra for seven red giant stars in the Boötes I dSph galaxy using standard abundance analysis techniques. In particular, we assume local thermodynamic equilibrium and employ spherical model atmospheres and codes that take the sphericity of the star into account when calculating the elemental abundances.

Results. We confirm previous determinations of the mean metallicity of the Boötes I dwarf spheroidal galaxy to be -2.3 dex. Whilst five stars are clustered around this metallicity, one is significantly more metal-poor, at -2.9 dex, and one is more metal-rich at, -1.9 dex. Additionally, we find that one of the stars, Boo-127, shows an atypically high $[\mathrm{Mg} / \mathrm{Ca}] \mathrm{ratio}$, indicative of stochastic enrichment processes within the $\mathrm{dSph}$ galaxy. Similar results have previously only been found in the Hercules and Draco dSph galaxies and appear, so far, to be unique to this type of galaxy.
\end{abstract}

Key words. galaxies: individual: Boötes I - stars: abundances - galaxies: abundances - galaxies: dwarf - Local Group

\section{Introduction}

Until recently, the number of dwarf spheroidal (dSph) galaxies around the Milky Way was small compared to expectations from $\Lambda C D M$ (Moore et al. 1999). However, in the past few years several new systems have been found through systematic searches (e.g. Belokurov et al. 2006, 2007).

In general, dSph galaxies are some of the most tenuous stellar systems that we know of. This is especially true for the newly found dSph galaxies which have very low stellar luminosities (see e.g., Martin et al. 2008). The new dSphs are ultra-faint and show low metallicities as indicated by low-resolution spectroscopy (Kirby et al. 2008; Koch 2009). Koch et al. (2008) found unusual abundance patterns in two red giant stars (RGB) in the ultra-faint Hercules dSph galaxy. Because of the low baryonic mass for these system it has been speculated that the elemental abundances in the stars in these systems might show us the results of individual supernova events (Koch et al. 2008).

The recently found $\mathrm{dSph}$ galaxy in Boötes (Boötes I, Belokurov et al. 2006) provides an excellent opportunity to

* The data presented herein were obtained at the W.M. Keck Observatory, which is operated as a scientific partnership among the California Institute of Technology, the University of California and the National Aeronautics and Space Administration. The Observatory was made possible by the generous financial support of the W.M. Keck Foundation. test whether or not unusual elemental abundance ratios are a common feature of ultra-faint dSph galaxies, thanks to its low baryonic mass, Belokurov et al. (2006) estimate, based on a colour magnitude diagram, that the Boötes I dSph galaxy is a purely old and metal-poor system. Low-resolution spectroscopic data confirm this (Martin et al. 2007; Norris et al. 2008) find $\langle[\mathrm{Fe} / \mathrm{H}]\rangle=-2.5$.

With $M_{\mathrm{V}} \sim-5.8$ this dSph galaxy is one of the least luminous galaxies known (Belokurov et al. 2006). Fellhauer et al. (2008), modelled the system and find that if this galaxy ever had a dark matter halo, it must still have it. This implies that, since the dark matter provides a deep potential well, the stars that originally formed in the dSph galaxy are still there and, moreover, the depth of the well should have helped retain the ejecta from core-collapse supernova. For Hercules, Koch et al. (2008) conclude that about 10 supernova are needed to pollute the interstellar medium to the observed atypical abundance ratios. Given that Boötes I has an even lower baryonic mass than Hercules, we might expect to be able to see enrichment from individual supernovae in the elemental abundance trends (which would show up as large scatter in element ratios from star to star).

We have obtained high-resolution, moderate $\mathrm{S} / \mathrm{N}$ spectra for seven RGB stars in the Boötes I dSph galaxy. Here we report on the mean metallicity, the metallicity spread, and atypical abundance ratios similar to those found in the Hercules (Koch et al. 2008) and Draco dSph galaxies (Fulbright et al. 2004). Thus, 
Table 1. Identification of programme stars and the derived elemental abundances.

\begin{tabular}{llllllllllll}
\hline \hline ID & $\begin{array}{l}\text { RA } \\
(\mathrm{J} 2000.0)\end{array}$ & $\begin{array}{l}\text { Dec } \\
(\mathrm{J} 2000.0)\end{array}$ & $V$ & $\begin{array}{l}{[\mathrm{Fe} / \mathrm{H}]} \\
(7.45)\end{array}$ & $\begin{array}{l}{[\mathrm{Fe} / \mathrm{H}]} \\
\text { Norris08 }\end{array}$ & $\epsilon(\mathrm{Fe})$ & $\epsilon(\mathrm{Mg})$ & & $\epsilon(\mathrm{Ca})$ & $\epsilon(\mathrm{Ba})$ & \\
\hline Boo-007 & 135935 & +142023 & 17.88 & -2.28 & -2.32 & $5.170 .26(22)$ & $5.680 .18(4)$ & 5.57 & $4.240 .22(5)$ & $-0.90(1)$ \\
Boo-033 & 140011 & +142501 & 17.79 & -2.47 & -2.96 & $4.980 .36(25)$ & $5.530 .30(4)$ & 5.77 & 4.22 & $0.28(5)$ & $-0.740 .24(2)$ \\
Boo-094 & 140031 & +143403 & 16.99 & -2.90 & -2.79 & $4.550 .22(25)$ & $5.140 .04(4)$ & 5.12 & 3.61 & $0.03(4)$ & \\
Boo-117 & 140010 & +143145 & 17.74 & -2.24 & -1.72 & $5.210 .30(21)$ & $5.660 .23(3)$ & 5.53 & $4.340 .28(4)$ & $-0.570 .11(2)$ \\
Boo-121 & 140036 & +143927 & 17.42 & -2.39 & -2.37 & $5.060 .22(26)$ & $5.530 .30(4)$ & 5.78 & $4.280 .29(5)$ & $-0.69(1)$ \\
Boo-127 & 140014 & +143552 & 17.69 & -1.98 & -1.49 & $5.470 .34(29)$ & $6.280 .11(3)$ & 6.33 & 4.33 & $0.18(5)$ & $-0.490 .43(2)$ \\
Boo-911 & 140001 & +143651 & 17.49 & -2.21 & -1.98 & $5.240 .29(29)$ & $5.630 .24(4)$ & 5.45 & 4.48 & $0.05(4)$ & $-0.640 .09(2)$ \\
\hline
\end{tabular}

Boötes I becomes the third system to show unexpected abundance ratios.

\section{Observations and abundance analysis}

Observations using the High Resolution Echelle Spectrometer (HIRES) (Vogt et al. 1994) on Keck I were carried out in June 2006. We obtained spectra of reasonable quality for seven RGB stars in the Boötes I dSph galaxy.

A full description of the construction of the linelist, determination of stellar parameters, etc. will be given elsewhere. Briefly, we derived $T_{\text {eff }}$ from infrared 2MASS photometry (Skrutskie et al. 2006) using the calibrations by Houdashelt et al. (2000) and Alonso et al. (1999). We found that all stars could be modelled using $T_{\text {eff }}=4600 \mathrm{~K}$, apart from Boo-094, which has a somewhat cooler model. Once $T_{\text {eff }}$ was determined, microturbulence $\left(\xi_{\mathrm{t}}\right)$ was checked by requiring all Fe I lines to yield the same Fe abundance regardless of linestrength. Given the quality of the spectra, a common $\xi_{\mathrm{t}}$ of $2.1 \mathrm{~km} \mathrm{~s}^{-1}$ yields a consistent result for all stars. Surface gravity $(\log g)$ was set to 1.0 dex for all stars, apart from Boo-094, for which we adopt 0.5 dex. Boo-094 is clearly more evolved; e.g., lines sensitive to $\log g$, such as the Ca I line at $616.2 \mathrm{~nm}$, show that this star has a low $\log g$. Our results are not sensitive to the adopted $\log g$ (compare Fig. 2).

Assuming that local thermodynamical equilibrium will hold, we performed a standard abundance analysis using MARCS model atmospheres (Gustafsson et al. 2008) and accompanying programs for abundance analysis. These codes take the sphericity of the stellar atmospheres into account, which is necessary because our stars are quite evolved. Using nonspherical models results in significant errors (Heiter \& Eriksson 2006). The calculations of the elemental abundances incorporate the broadening of the lines through collisions by neutral hydrogen (Barklem \& O’Mara 1997; Barklem et al. 1998; Barklem \& O'Mara 1998; Barklem et al. 2000; Barklem \& Aspelund-Johansson 2005).

We show two portions of stellar spectra in Fig. 1. Boo-094 clearly has the highest $\mathrm{S} / \mathrm{N}$ and the weakest spectral lines. That this star comes out as the most metal-poor in our abundance analysis is hence not surprising (cf. Table 1).

We find it entirely plausible that our $[\mathrm{Fe} / \mathrm{H}]$ determinations, and others', can have systematic and/or random errors as large as $0.3-0.5$ dex. We arrive at this conclusion based on results such as those shown in Fig. 2. In Fig. 2 we investigate how much the abundance ratios change when $T_{\text {eff }}$ is changed by $-200 \mathrm{~K}$ and $+150 \mathrm{~K}, \log g$ by $+0.5 \mathrm{dex}, \xi_{\mathrm{t}}$ by +0.6 and $-0.4 \mathrm{~km} \mathrm{~s}^{-1}$, and $[\mathrm{Fe} / \mathrm{H}]$ by -0.5 dex. Remarkably, $[\mathrm{Mg} / \mathrm{Ca}]$ hardly changes, whilst the greatest differences for $[\mathrm{Fe} / \mathrm{H}]$ amounts to 0.5 dex. However, even given the uncertainty in $[\mathrm{Fe} / \mathrm{H}]$, it appears very clear that Boo-094 is significantly more metal-poor than the bulk of RGB stars in the Boötes I dSph galaxy.

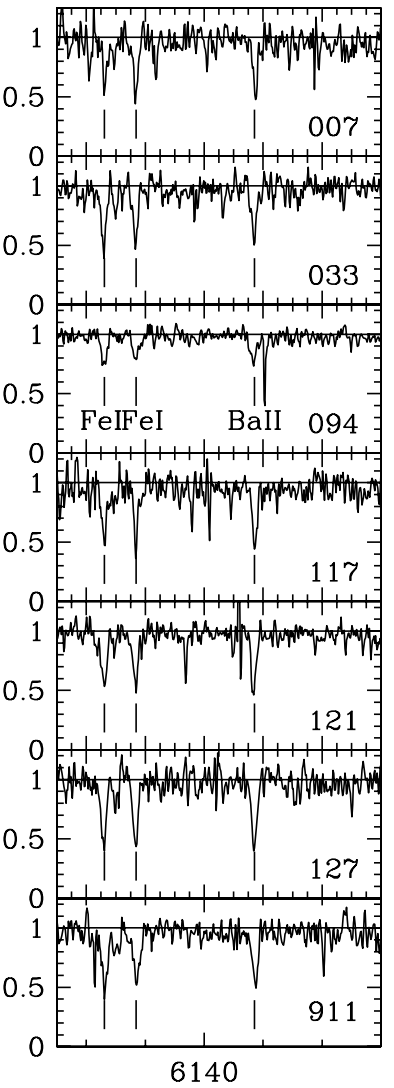

$\lambda(\AA)$

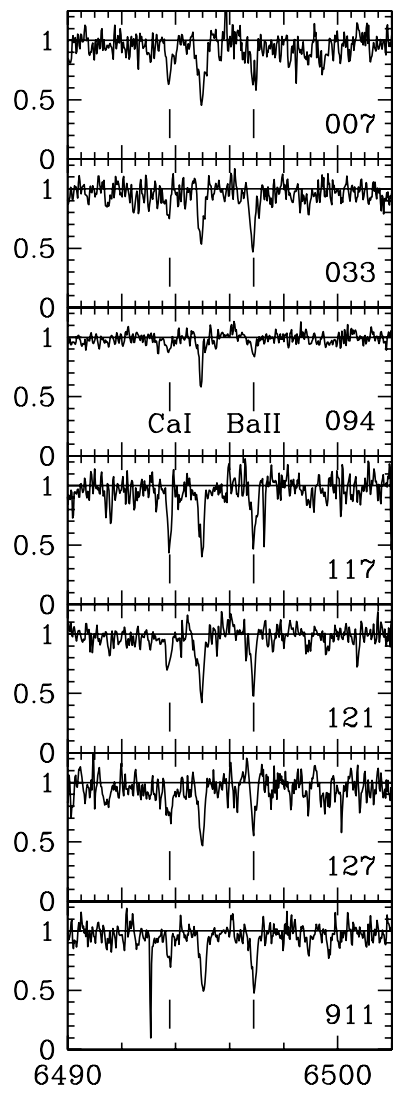

$\lambda(\AA)$
Fig. 1. Portions of the stellar spectra around the two Ba II lines used in this study. Those and additional lines measured are identified in the panels for Boo-094 (third from the top). $\mathrm{S} / \mathrm{N}$ are typically somewhat higher than 20-25. For Boo-094 S/N reaches 30-35.

From our sample of 7 stars we find a mean iron abundance of 5.1 , on the scale where $\log N(\mathrm{H})=12.00$, with a sigma of 0.3 dex. This gives an $[\mathrm{Fe} / \mathrm{H}]$ of -2.35 dex with the solar iron abundance taken to be 7.45 (Asplund et al. 2006). This is compatible with what has previously been found for the system (Norris et al. 2008). The determinations by Norris et al. (2008) are based on measurements of the $\mathrm{Ca} \mathrm{H}$ and $\mathrm{K}$ lines. The difference in $[\mathrm{Fe} / \mathrm{H}]$ is -0.1 dex with a sigma of 0.3 dex (cf. Table 1 ). This must be regarded as excellent agreement.

However, a deeper investigation reveals that there is a trend between our data such that, for low-metallicity stars, our $[\mathrm{Fe} / \mathrm{H}]$ are higher than those found in Norris et al. (2008). It is not clear where this difference stems from and our data-set is too small to investigate this further. We note, however, that the difference between us and Norris et al. (2008) also correlates with the equivalent width of the Ca II triplet line at $855.2 \mathrm{~nm}$ as 


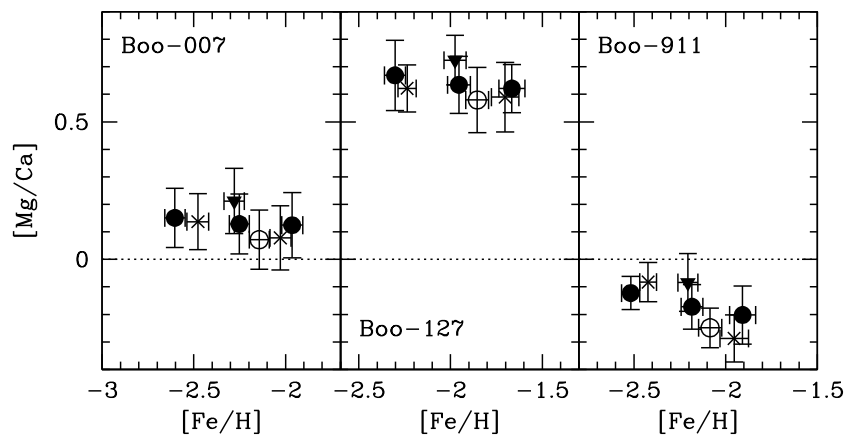

Fig. 2. Illustration of the robustness of the measured $[\mathrm{Mg} / \mathrm{Ca}]$ to errors in the stellar parameters. For each star we show the $[\mathrm{Mg} / \mathrm{Ca}] \mathrm{ra}-$ tio using seven model atmospheres all with different stellar parameters: $\bullet$ shows three models with different $T_{\text {eff }}$ (the middle one being the one adopted in the final abundance analysis). A filled, inverted triangle denotes a model with the final $T_{\text {eff }}$ but with $\log g=0.5$, and $\circ$ denotes a model with the finally adopted $T_{\text {eff }}, \log g=1.0$, and the finally adopted $\xi_{\mathrm{t}}$ but with $[\mathrm{Fe} / \mathrm{H}]=-2.50$. Finally, $\times$ denotes two models with all parameters set to the finally adopted values but with $\xi_{\mathrm{t}}+0.6$ and $-0.4 \mathrm{~km} \mathrm{~s}^{-1}$ relative to the final value. The error-bars denote the error in the mean for the derived abundances; e.g., for $[\mathrm{Mg} / \mathrm{Ca}]$ this is equivalent to $\sqrt{\sigma_{\mathrm{Mg}}^{2} / N_{\text {lines, } \mathrm{Mg}}+\sigma_{\mathrm{Ca}}^{2} / N_{\text {lines }, \mathrm{Ca}} \text {. }}$

measured by them, such that the difference is large and positive for smaller equivalent widths and large and negative for stronger lines. A more extensive comparison should be made to conclude on the source of the difference found.

Identifications and elemental abundances for our stars are listed in Table 1. The table contains the following in formation: column one lists the stars' designations as used in this paper and in Norris et al. (2008). Columns two and three list the right ascension and declination, respectively. Column four lists the $V$ magnitude. Columns five and six list our $[\mathrm{Fe} / \mathrm{H}]$ (assuming a solar $[\mathrm{Fe} / \mathrm{H}]$ of 7.45 ) and the $[\mathrm{Fe} / \mathrm{H}]$ derived by Norris et al. (2008). Columns seven, eight, ten, and eleven then list our derived abundances as indicated. For each element, we first list the abundance, the line-to-line scatter and, then the number of lines in parenthesis. For $\mathrm{Mg}$ (column nine) we give the $\mathrm{Mg}$ abundance if the two strong Mg triplet lines at 5172.6 and $5183.6 \AA$ are excluded.

\section{The metallicity (spread) in the Boötes I dSph galaxy}

It thus appears that the metallicity distribution, as derived from high-resolution spectroscopy, on the upper RGB of the Boötes I $\mathrm{dSph}$ galaxy is dominated by one metallicity with few outliers. That the outliers are real and not caused by measurement errors is further demonstrated by inspection of the stellar spectra (Fig. 1 and Sect. 2). The stars in our study span about 1 dex. Norris et al. (2008) find a total spread of about 1.7 dex and Martin et al. (2007) a spread of 1.3 dex. Both of these studies include more stars than our study.

The position on the sky of our stars do not indicate that, e.g., the most metal-poor star is at the outskirts of Boötes I. In fact, the overall shape and extent of the Boötes I dSph are currently poorly constrained. The radial velocity selected stars from Martin et al. (2007) and Norris et al. (2008) appear to have complementary sky coverage. All our stars are also studied by Norris et al. (2008), so are radial velocity members (see their Table 1).

\section{Abundance ratios - signs of individual supernovae?}

Koch et al. (2008) have observed stars in the Hercules $\mathrm{dSph}$ galaxy and found atypical abundance ratios. The Hercules dSph galaxy is one of the ultra-faint, newly discovered dSph galaxies (Belokurov et al. 2007; Adén et al. 2009). One star in Draco, a classical dSph galaxy, also shows this unusual abundance pattern (Dra-119 Fulbright et al. 2004). We find one star in the Boötes I dSph galaxy that clearly shows the same unusual $[\mathrm{Mg} / \mathrm{Ca}]$ pattern, Boo-127 (Fig. 3). It is also possible that Boo-094 shows similar traits but not as clearly.

It is clear that Her-2 and Her-3, Dra-119, and Boo-127 all stand out very clearly from the common trend. Even with large errors in the stellar parameters, Boo-127 cannot be made compatible with the general trend found for the other RGB stars in the Boötes I dSph galaxy. Note also that we have 3-4 Mg lines in our abundance analysis, while Koch et al. (2008) only used one. Our four lines show consistent and high $\mathrm{Mg}$ abundances (cf. Table 1). Thus our results confirm and strengthen the results found for the Draco and Hercules dSph galaxies.

Additionally, Koch et al. (2008) and Fulbright et al. (2004) find that Her-2, Her-3, and Dra-119, respectively, have extremely weak or nonexistent $\mathrm{Ba}$ II lines resulting in upper limits on the $[\mathrm{Ba} / \mathrm{H}]$ abundances. For the stars in the Boötes I dSph galaxy, this does not appear to be the case (compare Fig. 1). We have not derived Ba II abundances for Boo-094, because on closer inspection, the lines are not free from blemishes, but they are clearly visible in the stellar spectra.

However, for metal-poor stars, like Boo-094, the S/N of the spectrum is clearly important for a positive detection of the Ba II line at $649.689 \mathrm{~nm}$ (compare Fig. 1). A comparison with Fig. 2 in Koch et al. (2008) shows that it might be possible that the line is buried in the noise. Those spectra also have somewhat lower resolution. Koch (2009, priv. com.) confirms that this might be a possibility, but that it is unlikely that, if the line was present at a standard level, it could be completely veiled by the noise and lower resolution; hence, the Ba abundance would still be low. Higher resolution, higher $\mathrm{S} / \mathrm{N}$ spectra of the stars in the Hercules dSph are needed to fully settle the issue.

In supernova explosions, freshly synthesized elements are ejected into the nearby interstellar medium. The yields of different elements depend on the mass of the star that is the progenitor (e.g. Woosley \& Weaver 1995; Iwamoto et al. 1999). In models of galactic chemical evolution, it is often assumed that the ejecta from the supernova become well-mixed quickly. This is the instantaneous recycling approximation (e.g. Pagel 1997). This works well when we explore the later phases of galactic chemical evolution or study galaxies as a whole; however, in situations where only one or few supernova have had the chance to enrich the interstellar medium, the gas will not be well-mixed, and we might therefore see an atypical composition of elemental abundances in a single star (Karlsson \& Gustafsson 2005). Few studies have been done of the effect on elemental abundances in low mass systems such as the ultra-faint dSph galaxies. As opposed to the more robust predictions from models of galactic chemical evolution that concerns giant galaxies such as the Milky Way, models for dSph galaxies will be highly vulnerable to any uncertainties in the supernova yields used for the modelling. As such yields remain uncertain, we can only at this point speculate on the origin of the atypical abundance ratios observed.

Koch et al. (2008) speculate that, if the enrichment histories of the ultra-faint $\mathrm{dSph}$ galaxies are largely dominated by 


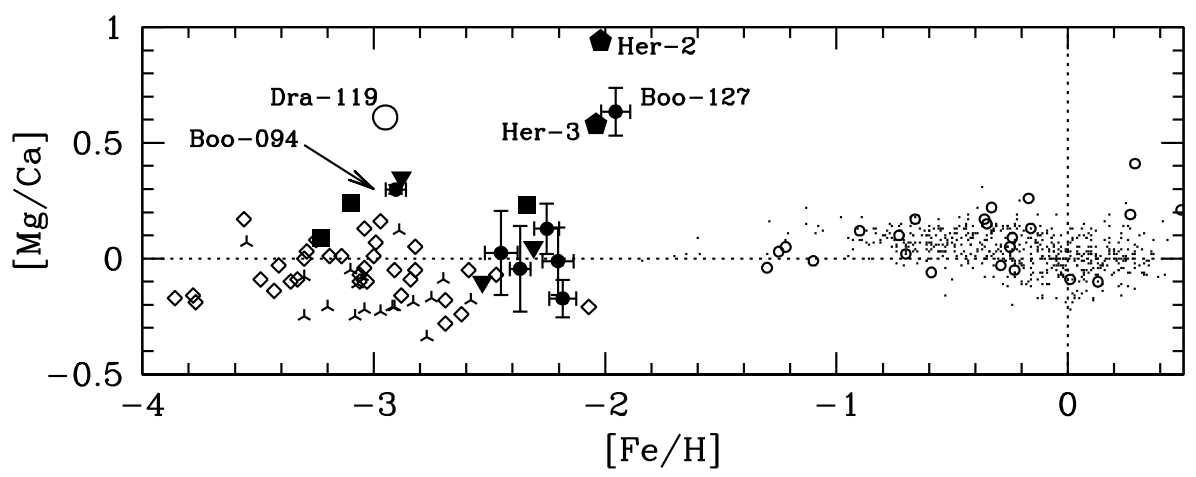

Fig. 3. $[\mathrm{Mg} / \mathrm{Ca}]$ as a function of $[\mathrm{Fe} / \mathrm{H}]$ for Boötes I (• with error-bars), Hercules (solid pentagons, Koch et al. 2008), Dra-119 ( $\bigcirc$, Fulbright et al. 2004), Ursa Major II (filled squares), and Coma Berenices (filled, inverted triangles) (both from Frebel et al. 2009). Bulge giants (o, Fulbright et al. 2007), Milky Way solar neighbourhood dwarf stars (·, Bensby et al., in prep.), halo giant stars (tripods, Cayrel et al. 2004), halo dwarf stars ( $\diamond$ Bonifacio et al. 2009).
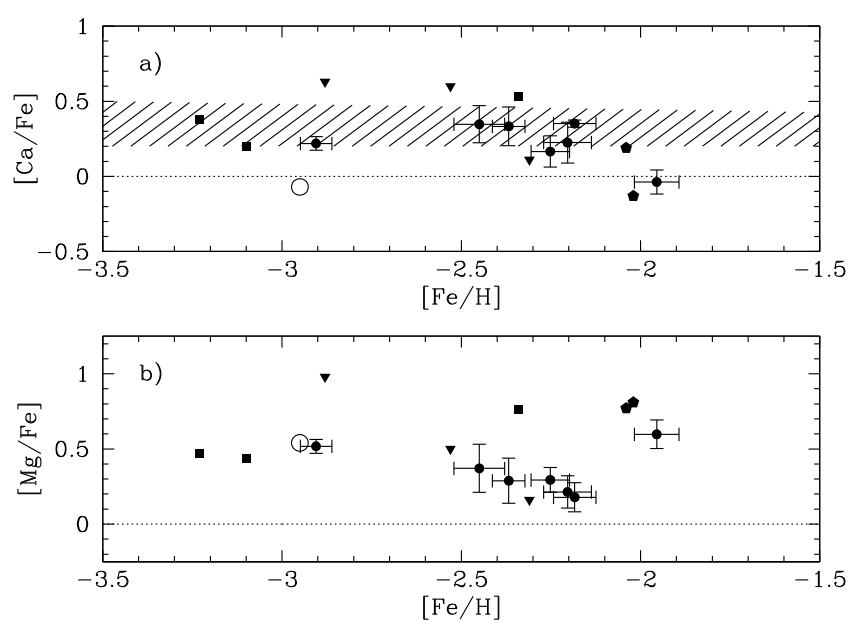

Fig. 4. a) A comparison of $[\mathrm{Ca} / \mathrm{Fe}]$ vs. $[\mathrm{Fe} / \mathrm{H}]$ for stars in several ultrafaint dSph galaxies. Boötes I (• with error-bars) Dra-119 ( $\bigcirc$ Fulbright et al. 2004), Hercules (filled pentagons, Koch et al. 2008), Ursa Major II (filled squares) and Coma Berenices (filled, inverted triangles) (both from Frebel et al. 2009). The hashed area shows the general trend for halo stars in the Milky Way. b) The same stars as in a) but for $[\mathrm{Mg} / \mathrm{Fe}]$ vs. $[\mathrm{Fe} / \mathrm{H}]$.

inhomogeneous evolution, considerable star-to-star scatter should be observed in these systems. Apart from Boo-127 (and possibly Boo-094), the remaining stars in Boötes I show considerable homogeneity in their derived $\mathrm{Mg}$ and $\mathrm{Ca}$ abundances (Fig. 4). Also for the two faint systems Coma Berenices and Ursa Major II, Frebel et al. (2009) find very homogeneous $[\mathrm{Mg} / \mathrm{Ca}]$ abundance ratios (Fig. 3) and fairly homogeneous abundance trends for the $\alpha$-elements (Fig. 4). These findings seem to suggest that the ultra-faint $\mathrm{dSph}$ galaxies are not, after all, different from the classical dSph galaxies. Indeed, Draco, a classical dSph, has one odd star (Fulbright et al. 2004), and the remainder are normal (Compare, e.g., the recent results in Cohen \& Huang 2009, which shows that eight of the bright red giants in Draco all have normal $\mathrm{Mg}$ to Ca ratios.).

Acknowledgements. S.F. is a Royal Swedish Academy of Sciences Research Fellow supported by a grant from the Knut and Alice Wallenberg Foundation. K.E. gratefully acknowledges support from the Swedish Research Council. M.I.W. is supported by a Royal Society University Research Fellowship. The authors wish to recognize and acknowledge the very significant cultural role and reverence that the summit of Mauna Kea has always had within the indigenous Hawaiian community. We are most fortunate to have the opportunity to conduct observations from this mountain. This publication makes use of data products from the Two Micron All Sky Survey, which is a joint project of the University of Massachusetts and the Infrared Processing and Analysis Center/California Institute of Technology, funded by the National Aeronautics and Space Administration and the National Science Foundation.

\section{References}

Adén, A., Feltzing, F., Koch, A., et al. 2009, A\&A, 506, 1147 Alonso, A., Arribas, S., \& Martínez-Roger, C. 1999, A\&AS, 140, 261 Asplund, M., Grevesse, N., \& Sauval, A. J. 2006, Nuclear Physics A, 777, 1 Barklem, P. S., \& O'Mara, B. J. 1997, MNRAS, 290, 102 Barklem, P. S., \& O’Mara, B. J. 1998, MNRAS, 300, 863 Barklem, P. S., \& Aspelund-Johansson, J. 2005, A\&A, 435, 373 Barklem, P. S., O’Mara, B. J., \& Ross, J. E. 1998, MNRAS, 296, 1057 Barklem, P. S., Piskunov, N., \& O’Mara, B. J. 2000, A\&AS, 142, 467 Belokurov, V., Zucker, D. B., Evans, N. W., et al. 2006, ApJ, 647, L111 Belokurov, V., Zucker, D. B., Evans, N. W., et al. 2007, ApJ, 654, 897 Bonifacio, P., Spite, M., Cayrel, R., et al. 2009, A\&A, 501, 519

Cayrel, R., Depagne, E., Spite, M., et al. 2004, A\&A, 416, 1117 Cohen, J. G., \& Huang, W. 2009, ApJ, 701, 1053

Fellhauer, M., Wilkinson, M. I., Evans, N. W., et al. 2008, MNRAS, 385, 1095 Frebel, A., Simon, J. D., Geha, M., \& Willman, B. 2009 [arXiv:0902 . 2395] Fulbright, J. P., Rich, R. M., \& Castro, S. 2004, ApJ, 612, 447

Fulbright, J. P., McWilliam, A., \& Rich, R. M. 2007, ApJ, 661, 1152

Gustafsson, B., Edvardsson, B., Eriksson, K., et al. 2008, A\&A, 486, 951 Heiter, U., \& Eriksson, K. 2006, A\&A, 452, 1039

Houdashelt, M. L., Bell, R. A., \& Sweigart, A. V. 2000, AJ, 119, 1448 Iwamoto, K., Brachwitz, F., Nomoto, K., et al. 1999, ApJS, 125, 439 Karlsson, T., \& Gustafsson, B. 2005, A\&A, 436, 879

Kirby, E. N., Simon, J. D., Geha, M., Guhathakurta, P., \& Frebel, A. 2008, ApJ, 685, L43

Koch, A. 2009, Rev. Mod. Astron., 21, 9, to appear [arXiv: 0905.0557]

Koch, A., McWilliam, A., Grebel, E. K., Zucker, D. B., \& Belokurov, V. 2008, ApJ, 688, L13

Martin, N. F., Ibata, R. A., Chapman, S. C., Irwin, M., \& Lewis, G. F. 2007, MNRAS, 380, 281

Martin, N. F., de Jong, J. T. A., \& Rix, H.-W. 2008, ApJ, 684, 1075

Moore, B., Ghigna, S., Governato, F., et al. 1999, ApJ, 524, L19

Norris, J. E., Gilmore, G., Wyse, R. F. G., et al. 2008, ApJ, 689, L113

Pagel, B. E. J. 1997, Nucleosynthesis and chemical evolution of galaxies (Cambridge: Cambridge University Press)

Skrutskie, M. F., Cutri, R. M., Stiening, R., et al. 2006, AJ, 131, 1163

Vogt, S. S., Allen, S. L., Bigelow, B. C., et al. 1994, in SPIE Conf. Ser. 2198, ed. D. L. Crawford, \& E. R. Craine, 362

Woosley, S. E., \& Weaver, T. A. 1995, ApJS, 101, 181 